\title{
EL CAMBIO TECNOLÓGICO EN LA INDUSTRIA MAQUILADORA DE EXPORTACIÓN EN MÉXICO: UN ENFOQUE METODOLÓGICO*
}

\author{
Por \\ J. Alberto Godínez Plascencia**
}

\begin{abstract}
RESUMEN
El presente trabajo hace una revisión crítica de los estudios más representativos que analizan el cambio tecnológico en la industria maquiladora en México (IMB). El objetivo es realizar una evaluación de los diferentes elementos que se estudian en cada uno de los trabajos, haciendo un énfasis especial en los instrumentos de medición y la base de datos.

$\mathrm{El}$ argumento que sustenta el trabajo es que la diversidad de resultados y conclusiones son producto de la misma heterogeneidad de las plantas maquiladoras, pero también de la diversidad de los objetivos, hipótesis, marco teórico, variables, indicadores, instrumentos de medición, base de datos, etc., que utilizan los estudios analizados.

Las conclusiones plantean la siguiente pregunta: Existe un cambiode carácter estructural en el nivel tecnológico de la IME? Actualmente, en la IMB existe una heterogeneidad en la base tecnológica que se manifiesta en una dualidad tecnológica, que se mantiene como estructura y como proceso tendencial. Este esquema se reproduce tanto entre las plantas como al interior de las mismas que cuentan con tecnologías y formas de organización más avanzadas. Además, este apartado proporciona una serie de recomendaciones técnico-metodológicas, con el fin de mejorar y eficientar los instrumentos de medición y base de datos sobre el cambio tecnológico u otras variables que se deseen conocer para la MB.
\end{abstract}

\section{ABSTRACT}

This paper gives a critical review of one of the most representative studies that analyse the tecnhological change of the maquiladora industry in Mexico.

The objective is to assess the different elements studied in each work, with a special emphasis in the measuring instruments and the data base.

The argument supporting this article, is that the diversity of the issues and conclusions are the result of the heterogeneity of the maquila plants, but also arise from the diversity of objectives, hypotheses, theories, variables, indicators, measuring instruments, data base, etc., that are used in the analized studies.

The conclusions pose a new question: is there really a structural change in the technological level in maquiladora industry of Mexico? Presently there exists a heterogeneous technological base in the maquiladoraindustry of Mexico, which is expressed in technological duality, both as structure and tendencial process. This scheme of duality occurs both among the plants as well as in the internal structure of individual maquila plants with better technology and organization. The conclusions provide some technical-methodological recomendations with the purpose of improving the measuring instruments and data base about technological change and variables in the maquiladora industry of Mexico.

* Agradezco la inspiración y el apoyo moral que me ha brindado Norma Violetta.

** Profesor-investigador del Departamento de Estudios Económicos del COLRF. 


\section{INTRODUCCIÓN}

...La forma más segura de condenar cualquier investigación es compararla con los ideales de la ciencia. La mejor manera de evaluar un trabajo de investigación es compararlo con olros, los más repulados dentro de nuestro campo (Zellerberg, 1965).

La mayoría de los estudios más recientes que han tenido como objeto de estudio a la industria maquiladora de exportación en México (IME). coinciden en señalar que esta industria ha entrado, a partir de la última década, en una serie de cambios estructurales que están dando como resultado una industria maquiladora cualitativamente diferente a la tradicional maquiladora de los años sesenta y setenta. Dentro de la serie de cambios estructurales se resalta el cambio en el nivel tecnológico. Se argumenta que la tecnología sencilla de ensamble manual está siendo sustituida por las categorías de la tecnología flexible como es la tecnología dura (maquinaria programable, diseños y manufactura asistida por computadora, robots industriales, etc.) y la tecnología blanda (nuevas técnicas de organización del trabajo y de la producción intraempresa e interempresas).

La mayoría de los estudios señalan la existencia de un cambio estructural en el nivel tecnológico. Sin embargo, existen otros que argumentan que si bien ha habido introducción de las nuevas tecnologías no es momento todavía como para hablar de un "cambioestructural". Aun dentro los estudios que sostienen la tesis de cambio tecnológico estructural, existe una diversidad de matices y posiciones. Sobresale la posición que afirma que el cambio estructural no se está dando de manera generalizada y homogénea, sino más bien de forma concentrada y heterogénea, tanto entre ramas y entre plantas como también en las plantas que han incorporado los avances tecnológicos y organizativos.

En este trabajo se argumenta que la diversidad de resultados y conclusiones es producto, en primera instancia, de la misma heterogeneidad de las plantas maquiladoras, pero también de la diversidad de elementos en que se basan cada uno de los estudios (objetivos, hipótesis, marco teórico, variables, indicadores, instrumentos de medición, base de datos, etc.). De esta forma, el objetivo principal del trabajo consiste en realizar una revisión crítica de estos elementos para cada uno de los estudios seleccionados, haciendo especial énfasis en los instrumentos de medición y la base de datos.

Para centrar exclusivamente el análisis sobre los instrumentos de medición y las evidencias aportadas se trató de seleccionar aquellos estudios que sobresalen por su aportación y cuyos objetivos e hipótesis explícitas tuvieran que ver con la cuestión tecnológica en la IME. 
La revisión se hará sobre la base de las siguientes preguntas: 1) ¿Por qué ha cobrado tanto interés la cuestión tecnológica en la IME? interés manifestado en la proliferación de estudios con objetivos e hipótesis similares sobre el cambio tecnológico, 2) ¿Cuál es la cobertura tempo-espacial y confiabilidad del instrumento de medición y la base de datos?, 3) ¿Qué tan generalizables y sustentables son los resultados y conclusiones a las que se arriban? $\mathrm{y}, 4$ ) finalmente, a manera de conclusiones, ¿existe cambio tecnológico estructural en la IME?

En la primera parte se reseñan los principales cambios estructurales observados en la industria maquiladora, haciendo especial énfasis en el cambio tecnológico. En la segunda parte se analiza brevemente el marco teórico, objetivos e hipótesis. En la tercera se centra la atención en la confiabilidad y cobertura tempo-espacial de los instrumentos de medición y la base de datos utilizados para respaldar los resultados y las conclusiones. Finalmente, en las conclusiones se tratará de dar respuesta en forma global a la pregunta: $\iota$ existe un cambio tecnológico de corte estructural en la industria maquiladora de exportación en México?

\section{CAMBIO TECNOLÓGICO ESTRUCTURAL}

Formalmente la IME en México dio inicio en 1965 en las ciudades de la frontera norte bajo el Programa de Industrialización Fronteriza. Es decir, se adopta un esquema explícito para participar en la subcontratación internacional de servicios productivos cuyo objetivo es completar en forma rápida, fluida y barata las últimas fases del proceso productivo iniciado en otro país. Así, hasta antes de la década de los ochenta, las unidades económicas de la IME son básicamente concebidas como plantas con una tecnología sencilla, procesos cortos de ensamble, intensivas en mano de obra y con una gran facilidad de relocalización geográfica. Bajo estas características la IME ha resultado ser un éxito en cuanto al crecimiento de las principales variables económicas. Baste decir que de haber en 1966 sólo 12 plantas que daban empleo a 3,107 personas, para 1990 el número de plantas ascendió a 1,938 y emplearon 460,293 personas. El crecimiento medio anual para ambas variables fue de $23.6 \mathrm{y}$ $23.2 \%$ respectivamente. El valor agregado en 1974 , medido a valor constante de ese mismo año, fue de 3,945 millones de pesos mientras que para 1990 fue de 21,732 , por lo que la tasa de crecimiento resultó ser del $11.3 \%$ (INEGI, 1991).

Si los cambios cuantitativos han sido sorprendentes, lo son aún más los cambios cualitativos que han empezado a observarse más claramente a partir de la segunda mitad de los ochenta y que, a partir de la literatura 
consultada, pueden resumirse en los siguientes puntos ${ }^{1}: 1$ ) cambio en la composición sectorial, donde las ramas tradicionales como la textil, la del vestido, el calzado, la alimenticia y muebles han cedido su importancia económica a la rama eléctrica, electrónica y automotriz; 2 ) concentración de la producción y el empleo en las grandes empresas multinacionales de origen norteamericano y recientemente de origen asiático; 3) cambios en la naturaleza del proceso de producción. Con respecto a este punto es necesario resaltar cuatro aspectos: a) las líneas cortas y sencillas de producción se están sustituyendo por líneas más largas y complejas que se asemejan cada vez más a un proceso de manufactura; b) la tecnología sencilla del ensamble manual estả siendo desplazada por la introducción de los principales componentes de la automatización flexible como son las máquinas de control numérico y los robots industriales; c) introducción de las nuevas técnicas de organización interna del trabajo como los circulos de calidad y "justo a tiempo" y d) la conformación de "centros regionales de producción" donde las grandes empresas maquiladoras filiales de las multinacionales organizan a su alrededor geográfico a sus principales proveedores y/o subcontratistas y usuarios finales.

Sin lugar a dudas tratar de identificar y explicar las causas, condiciones e implicaciones de los cambios estructurales de la IME es una tarea multidisciplinaria, amplia y compleja quedesborda los límites y objetivos del presente trabajo. Sólo interesa rescatar el consenso que afirma que se está presenciando la formación y consolidación de una industria maquiladora esencialmente nueva y diferente a la existente hace tan sólo 10 años, y una de las principales características de la nueva maquiladora es un nivel tecnológico sensiblemente más elevado y complejo. Pero si bien ese es el consenso, ¿cuáles son las posiciones contrarias? y ¿en qué sustentan sus afirmaciones cada una de las posiciones?

\section{MARCO TEÓRICO, OBJETIVOS E HIPÓTESIS}

\section{...no es posible evaluar la mayoría de los métodos prescindiendo de sus objetivos y de su propósito. (Harvey, 1969)}

En el cuadro 1 se presentan las hipótesis y objetivos planteados por los principales autores y estudios que han versado sobre los cambios tecnologicos en la IME. La selección de los estudios se realizó bajo el criterio de que, directa o indirectamente, se plantearan como objetivo analizar el

1 Una clasificación y análisis de las posibles causas de los cambios estructurales en la IMB es realizada por J. Carlos Ramírez y Bernardo González-Aréchiga (1989). 
cambio tecnológico y sus implicaciones para ciertas variables o características de la IME; y que se plantearan como hipótesis la existencia de un cambio estructural en la naturaleza y funcionamiento de la IME resaltando el cambio en el nivel tecnológico y organizativo.

El estudio teórico-empírico de Sanderson es pionero en plantearse como objetivo el análisis de los efectos de la introducción de las nuevas tecnologías en la naturaleza y funcionamiento de la industria maquiladora en México (Sanderson, 1987:127-148). Alternada y/o simultáneamente, y hasta la fecha, han proliferado estudios que han abordado el tema generando más conocimientos y aportando más riqueza a la discusión. Dentro de los autores resaltan las investigaciones de Bernardo González-Aréchiga (1989:16-29) y J. Carlos Ramírez (1989:97-150 y 1990:17-46) que no sólo han realizado estimaciones de la magnitud y dirección de los cambios estructurales y tecnológicos de la IME, sino que han tratado de enfocar el fenómeno de la subcontratación internacional en México en una dimensión más amplia y dinámíca, es decir, no circunscribirse al análisis estático y tratar de encontrar una unidad de análisis más precisa ya que la planta maquiladora $-\mathrm{y}$ el programa maquilador - cada vez es una unidad de análisis menos precisa y explicativa de la subcontratación internacional en nuestro país.

La proliferación de estudios con objetivos e hipótesis similares es el resultado de la necesidad apremiante de aportar evidencias empíricas al agudo debate teórico de corte intemacional, surgido a inicios de los ochenta, acerca de los efectos de las nuevas tecnologías sobre el proceso de redespliegue industrial (IME) de los países industrializados a los no industrializados.

En este debate se identifican claramente dos posiciones un tanto contradictorias. Por un lado, Rada (1983); Junne (1986) y Kaplinsky (1988:139-160) encabezan la tesis de que con el proceso ampliado de aplicación de las nuevas tecnologías al proceso de producción industrial, es de preveer un "regreso a casa" de las empresas multinacionales que, buscando la ventaja relativa de los bajos costos salariales, se ubican en algunos países no industrializados. Por el otro, Ernst encabeza la tesis contraria al afirmar que tal "regreso a casa" no es la tendencia predominante, sino que siguen ubicándose más empresas multinacionales en estos países, pero ahora sobre nuevos factores explicativos. Además, agrega que "...la automatización y el redespliegue industrial hacia los paises no industrializados no deben considerarse como procesos mutuamente excluyentes sino como procesos complementarios, con la automatización convirtiéndose cada vez más en la fuerza impulsora" (Emst, 1984:83-114). 


\section{CUADRO 1. Objetivos e hipótesis de los principales estudios sobre el cambio tecnológico en la industria maquiladora.}

$\begin{array}{lll}\text { Autor Objetivo(s) Hipótesis } & \end{array}$

Sanderson Este trabajo se centra sobre la industria electrónica y 1987 explora los efectos potenciales de las tecnologías de manufactura sobre las plantas maquiladoras en México y las implicaciones de política de estos cambios para México y Estados Unidos.

González Estimar los cambios en la tecnología, los patrones 1988 emergentes de protección e intercambio internacional en la IME y la organización de la producción.

Gonzálezy Estimar los incrementos no observables en el capital Ramírez por trabajador y en la productividad del trabajo.

$1988 \quad$ Medir la magnitud real de los cambios tecnológicos y organizativos del conjunto de la industria e identificar la dirección de los cambios.

Mertens y Investigar si el proceso de trabajo se transforma de Palomares manera significativa con la aplicación de la microelec-

1988 trónica en el proceso de producción.

Wilson . Examinar conceptual y empíricamente la naturaleza de 1989 la nueva maquiladora y su papel.
El trabajo directo esta comenzando a ser menos importante en los costos totales de muchas manufacturas, a la vez que se incrementa el uso de los diseños y manufactura ayudados por computadora (CAD/CAM) y robots industriales. Esto puede erosionar la ventaja comparativa de las regiones manufactureras de bajos salarios como es el caso de México.

Los cambios estructurales de laIME constituyen la base de un ajuste cuantitativo que refuerza su estado tradicional de dualismo tecnológico.

Está emergiendo un nuevo tipo de industria maquiladora de exportación (IME), físicamente más productiva que siendo altamente tecnificada, incorpora procesos completos de manufactura, pero que no refleja su productividad en mayor valor agregado y en aumento de los salarios.

Con la aplicación de los avances de la microelectrónica al proceso de producción de la industria electrónica, está surgiendo un nuevo tipo de proletariado.

Existe un cambio drástico en la naturaleza de la maquiladora desde inicios de los ochenta. Ahora se encuentran plantas manufactureras que han adaptado las nuevas tecnologías. 


\section{Brown y}

Domínguez

1989

Carrillo

1989

Godinez

1990

Mungaray

1990

González y

Ramírez

1990
Estudiar el proceso de incorporación de las nuevas tecnologías en la industria maquiladora.

Analizar las modalidades específicas de incorporación, sus determinantes y entender sus límites.

Estudiar los efectos en el empleo, en la composición de la fuerza de trabajo, en el perfil del trabajador demandado y en el proceso de trabajo.

Profundizar sobre el factor humano en el trabajo, a través de la percepción de los trabajadores directos hacia las nuevas tecnologías.

Clasificar y cuantificar el cambio tecnológico en la IME. Cuantificar el efecto del cambio tecnológico sobre la generación de empleo.

Analizar el comportamiento de distintas variables económicas que influyen o se relacionan con la productividad y la ganancia.

Explorar las consecuencias a largo plazo de las tendencias actuales y posibles sobre las principales variables descriptivas de la estructura y operación de la IME en su contexto amplio, tratando de escapar a las limitaciones propias de los análisis estáticos.
Se desplazará a la túpica empresa maquiladora de las etapas anteriores.

El desarrollo de la industria maquiladora está en una etapa caracterizada por un gran dinamismo, por el uso creciente de tecnologías flexibles y por los cambios en el perfil socio demográfico de los trabajadores.

Existe una percepción consensual en las plantas maquiladoras automotrices en relación con las viejas plantas no-maquiladoras.

A partir de 1982 se está dando un cambio estructural en el nivel tecnológico del proceso de producción de la IME. El cambio tecnológico es del tipo ahorrador de mano de obra.

En algunas actividades de maquila es necesario utilizar la alternativa de producción que ahorra costos de mano de obra combinados con el incremento en la productividad a través de mejoras tecnológicas.

En México la IME está en una fase de rápido crecimiento que la reorganiza y redefine, sin embargo, se mantiene arraigada al modelo maquilador tradicional, sólo que con un carácter estructural diferente y propio (dual, desintegrado y concentrado).

\section{FUENTE: Elaboración propia en base a la revisión de literatura teórica y empírica sobre la maquiladora.}


Sin embargo, debe seffalarse que ambas posiciones coinciden en seffalar que los salarios bajos de los países no industrializados están dejando de ser el factor principal del redespliegue.

En realidad, explícita o implícitamente, los objetivos e hipótesis planteadas por los estudios y/o autores del cuadro 1 tienen como referencia la discusión teórica de inicios de los ochenta, pero sobre todo tienen la preocupación de observar, medir, explicar y pronosticar los cambios en la naturaleza y funcionamiento de la industria maquiladora en México ante el proceso de incorporación de las nuevas tecnologias.

En síntesis, y por la revisión de las hipótesis planteadas, puede decirse que se converge en dos puntos principales: el primero, en el cual actualmente no existe discusión, es que el rápido crecimiento de las principales variables económicas de la IME en México es una evidencia que contradice la tesis del "regreso a casa"; el segundo, y en el cual existe todavía cierta discusión, es que en la IME se está dando un amplio y acelerado proceso de introducción de nuevas tecnologías y formas de organización del trabajo, al grado de que se puede hablar de un cambio de carácter estructural en el nivel tecnológico.

\title{
INSTRUMENTOS DE MEDICIÓN Y BASE DE DATOS
}

\begin{abstract}
...el fin de la inferencia inductiva es construir un lenguaje en el que se formalicen en reglas los pasas intuitivos. Estas reglas se encargan de asegurar que cualquiera que parta del mismo conjunto de hipótesis y las mismas evidencias llegará a la misma conclusión (Harvey, 1969).
\end{abstract}

Hasta aquí se ha visto que la mayoría de los estudios parten aproximadamente del mismo conjunto de hipótesis y se plantean objetivos similares. A continuación se analizará cómo se enfrenta la demostración de las hipótesis y el cumplimiento de los objetivos que se plantearon los estudios seleccionados. Para ello, en este apartado se centra la atención en revisar la confiabilidad y cobertura tempo-espacial y sectorial de los instrumentos de medición y la base de datos utilizados.

Ảl revisar los instrumentos de medición y la base de datos (ver cuadro 2) se llega a la conclusión de que existe una diversidad de instrumentos de medición. Sin embargo, se identifica una diversidad mayor en el tipo de evidencia empírica o base de datos presentada. ${ }^{2}$

2 El hecho de que una base de datos contenga algón elemento distinto de los que sa enumeran enseguida, constituye una evidencia empírica distinta: 1) población objetivo, 2) metodología, 3) tiempo de levantamiento, 4) continuidad y cobertura temporal, v) cobertura geogrifica y 6) cobertura sectorial. Se supone que la unidad de análisis para cualquier base de datos es la misma, es decir, la planta maquiladora. 


\section{Base de datos}

Recurriendo a los criterios de cobertura tempo-espacial y sectorial, nivel de desagregación, representatividad y pertinencia de las variables, se pueden clasificar las bases de datos en dos grupos bien definidos: el primero está constituido por las estadísticas oficiales que son levantadas y publicadas en forma mensual y/o anual por el Instituto Nacional de Estadística Geografía e Informática (INEGI); y el segundo lo constituyen las muestras que algunos investigadores levantaron directamente tratando de recoger y estimar variables e indicadores más pertinentes a la particularidad y objetivos del estudio.

\section{a) Estadísticas oficiales}

Definitivamente que cuando se habla del desempeño económico logrado por la IME en México se recurren a las estadísticas oficiales, principalmente a las variables de valor agregado, personal ocupado (empleo) y número de establecimientos. ${ }^{3}$ Pero en cuanto a variables e indicadores que midan y determinen la magnitud y dirección del cambio tecnológico, estas estadísticas proporcionan escasa información. ${ }^{4}$ En ese sentido no existe una pertinencia en las variables, es decir, una correspondencia directa entre el objetivo (medir el cambio tecnológico) y lo que representan y miden.

Dado que las estadísticas oficiales son el resultado de un censo, entonces la información tiene cobertura del total de las plantas registradas en el periodo del levantamiento, por lo que se supone que no deben existir problemas de representatividad.

Con respecto a la cobertura tempo-espacial se puede decir que prácticamente son las únicas estadísticas con continuidad en el tiempo. Desde 1974 y hasta la fecha, sistemáticamente se han levantado estadísticas en forma mensual y/o anual. Lo mismo ocurre con respecto a la cobertura

3 Las mismas estadísticas muestran el rotundo fracaso de tratar de integrar la IME con la industria local y/o nacional. En 1990 la industria maquiladora tan sólo utiliź el $1.7 \%$ de insumos nacionales del total de insumos (véase INEGI, 1990).

Las estadísticas oficiales proporcionan la desagregación de la variable "personal ocupado" en obreros, técnicos y administrativos. Esto permite la creación del indicador "técnicos/obreros" que arroja cierta información sobre ls introducción de las nuevas tecnologlas y formas de organización. Ya que se supone que las nuevas tecnologías dernandan znás personal calificado en comparación con el no calificado.

Más adelante se verá que, aun a pesar de la falta de pertinencia de las variables de las estadísticas oficiales, mediante el uso de una serie de modelos microeconométricos es posible estimur un conjunto de indicadores que arrojan suficiente luz como para clasificar y medir el cambio teconológico. 
espacial, prácticamente son cubiertas las principales ciudades y/o municipios del país donde se localice este tipo de industria tratando de diferenciar la rama industrial a la cual pertenece la información generada.

Al evaluar el nivel de desagregación, este tipo de estadísticas dejan mucho que desear en cuanto a proporcionar información a nivel de planta. El nivel menor de desagregación sectorial se queda hasta el nivel de rama industrial. Sin embargo, el mismo nivel de desagregación sectorial no se mantiene en un corte espacial a nivel de municipio, sino que se presentan los agregados totales para la industria.

Al observar el cuadro 2 se desprende que existe una relación directa entre los estudios que tomaron como base de datos las estadísticas oficiales, y los que utilizaron instrumentos económico-matemáticos para estimar un conjunto de indicadores sobre el cambio en la magnitud, dirección y tipo de cambio tecnológico de la IME. Con tales medios se trata de salvar la falta de pertinencia de las variables de las estadísticas oficiales.

\section{b) Muestras específicas}

Dados los inadecuados niveles de desagregación, la no pertinencia de las variables y la falta de información de las estadísticas oficiales acerca del proceso de incorporación de las nuevas tecnologías y formas de organización en el proceso productivo de la industria maquiladora, algunos estudiosos han tratado de corregir estas deficiencias en la información, recurriendo al levantamiento de muestras especiales que arrojen evidencias empíricas más pertinentes, precisas y ajustadas a los objetivos e hipótesis planteadas. Sin entrar a la revisión exhaustiva y cuidadosa de las variables e indicadores de los estudios del cuadro 2 que utilizaron muestras específicas, puede suponerse a grandes rasgos que se cumplen adecuadamente con los criterios de pertinencia y nivel de desagregación. Sin embargo, este tipo de muestras deja mucho que desear con respecto al resto de los criterios de evaluación.

Puede decirse que la principal debilidad de los estudios que utilizaron muestras específicas es la carencia de representatividad ${ }^{6}$ de la información, resultados y conclusiones. Es decir, los resultados y conclusiones obtenidas no pueden ser generalizados al universo o población objetivo. Son válidas exclusivamente para las plantas maquiladoras incluidas en la muestra. La única muestra con carácter representativo es la utilizada por

6 Por muestra representativa debe entenderse aquella que haya seguido alguna de las técnicas de muestreo aleatorio para garantizar la generalización y el grado de confiabilidad de los resultados. 
Bernardo González-Aréchiga (1989) y J. Carlos Ramírez (González-Aréchiga y Ramírez, 1989:97-160), ya que dicha muestra se refiere al total de plantas maquiladoras de la rama electrónica ubicada en Tijuana, B.C. en enero de 1988 (ver cuadro 2). El resto de las muestras, además de no ser representativas, se basaron en una selección dirigida bajo ciertos criterios (muestreo intuitivo) de las plantas que formarían la muestra.

Mientras más información se tenga sobre la naturaleza de las interacciones en la población, más fácil será elaborar métodos de muestreo adecuados $-\mathrm{y}$ aquí el enfoque del estudio de casos (muestreo intuitivo) tiene mucho que ofrecer como mecanismo exploratorio-. Se acepta generalmente que unas pocas observaciones bien elegidas pueden aportar tantas pruebas para la hipótesis como un enorme número. Lo importante es disponer de un método de recoger información que asegure la máxima independencia entre el método de toma y observación de datos y la hipótesis propuesta?

El muestreo aleatorio, al organizar la selección de observaciones en torno a un sistema puramente aleatorio, asegura la independencia entre el método de toma de datos y la hipótesis planteada. El muestreo intuitivo no puede utilizarse así, pero, no obstante, tiene una utilidad considerable, ya que permite examinar detenidamente las interacciones y las relaciones.

Aun suponiendo que las muestras específicas cumplan con el requisito de pertinencia, representatividad e independencia de la hipótesis con el método de toma de datos, quedan todavía por salvar los problemas de cobertura tempoespacial y sectorial.

Prácticamente todas las muestras específicas utilizadas por los estudios del cuadro 2 sucumben ante el requisito de la continuidad temporal, Es decir, no existen muestras que puedan ser comparables en el tiempo.

Por el tamaño de la muestra $y$, sobre todo, por la cobertura geográfica resalta el estudio de Mertens y Palomares (1985:170-198) que encuestó a 30 plantas electrónicas no maquiladoras y 30 plantas electrónicas maquiladoras en un amplio conjunto de ciudades del país. En el mismo sentido, también debe mencionarse el estudio de Patricia A. Wilson (1989) que, además, trata de incluir una variedad de plantas pertenecientes a las diferentes ramas industriales de la IME.

7 En la selección de las plantas maquiladoras que constituirán la muestra intuitiva, por lo general se incluyen precisamente las plantas maquiladoras más grandes y con mayores avances en cuanto a la introducción de las nuevas tecnologías y formas de organización. De esta forma es previsible esperar cuáles serán los resultados y conclusiones. Es decir, es dudoso que se mantenga la máxima independencia entre el método de toma de datos y la $(\mathrm{s})$ hipótesis planteada. 


\section{CUADRO 2. Instrumentos de medición y base de datos de los estudios sobre el cambio tecnológico en la IME.}

$\begin{array}{lll}\text { Autor Instrumento de medición } & \text { Base de datos }\end{array}$

Sanderson Desarrollo de un modelo costo-unitario para comparar 1987 las maquiladoras de ensamble manual con las de ensamble de automatización flexible.

Entrevista con diez gerentes, 25 trabajadores y 12 funcionarios públicos, promotores y representantes de organizaciones industriales.

González Aplicación de modelos microeconométricos 1988 Análisis de varianza (ANOVA).

González y Estimación indirectademodelosmicroeconométricos. Ramírez Análisis de varianza (ANOVA).

1988

Mertens y Palomares 1988 .
Visita y aplicación de un cuestionario a las empresas electrónicas (maquiladoras y no maquiladoras), ubicadas en la Ciudad de México, Puebla, Toluca, Guadalajara, Torreón, Monterrey, Reynosa, Nuevo Laredo, Chihuahua, Hermosillo, Agua Prieta, Nogales, Mexicali, Ensenada y Tijuana.
El modeloy las entrevistas corresponden a la frontera Tijuana-San Diego entre 1983 y 1984.

La encuesta es dirigida y no probabilística.

Serie de estadísticas básicas de la IME de 1980 a 1986 . INEGI.

Censo de las 257 empresas electrónicas de Tijuana levantado en 'enero de 1988 por el Depto. de Estudios Económicos del COLEF y SECOF. La base de información son los registros de SECOFI del Programa Maquilador.

Serie de estadísticas básicas de la IME de 1980 a 1986. INEGI.

Censo de las 257 empresas electrónicas de Tijuana levantado en enero de 1988 por el Depto. de Estudios Económicos del COLEF y SECOFL La base de información son los registros de SECOFI del Programa Maquilador.

Muestra dirigida (no probabilística) a 30 empresas productoras de computadoras y periféricos para el mercado interno y 30 empresas productoras de componentes, partes, subensambles y productos finales mayoritariamente para el mercado extemo y bajo el régimen maquilador. 
Wilson Aplicación de una encuesta dirigida a los gerentes

1989 generales o de producción de las plantas maquiladoras. La aplicación se realizó a finales de 1988 y principios de 1989.

Brown y Entrevista (encuesta no probabilística) a empresas.

Domínguez

1989

Carrillo

1989

Godínez

1990

Mungaray

1990

González y Ramírez 1990 rado en Marx, reelaborado con leves ajustes del que hicieran Ramos y Valle (1983), que tiene la gran virtud de desechar el supuesto pedagógico de la tasa de excedente constante que Marx utilizaba para mostrar la tendencia descendente de la tasa de ganancia.

Revisión de literatura teórica y estimaciones hechas por otros estudios y/o autores.
Encuesta dirigida a 71 plantas maquiladoras ubicadas en dos ciudades del interior (Monterrey y Guadalajara) y tres ciudades de la frontera norte (Tijuana, Ciudad Juárez y Nuevo Laredo).

Datos generados de entrevistas a las 20 empresas maquiladoras (electrónicas y de autopartes) más importantes de Ciudad Juárez.

Datos generados de los obreros directos de dos plantas de una misma firma automotriz. Una es maquiladora fronteriza y otra es terminal del centro-sur. Además se complementó con una muestra dirigida de 12 plantas maquiladoras de Cd. Juárez y Nvo. Laredo.

Estadísticas de la IME de 1974 a 1988 . La serie se subdividió en dos periodos (1974-1982 y 1982-1988) para tratar de observar en el tiempo los "cambios estructurales" en el nivel tecnológico.

Series históricas de las estadísticas básicas de la IME y del INEGI.

Modelo simplificado de excedentes y ganancias inspi-
Revisión de literatura teórica y estimaciones hechas por otros estudios $\mathrm{y} / \mathrm{o}$ autores.

FUENTE: Elaboración propia en base a la revisión de literatura teórica y empírica sobre la maquiladora. 
Sectorialmente, las muestras se concentran en las ramas electrónicas y de autopartes. Esto es así debido a que se supone que son las ramas en donde más rapidamente se están incorporando los avances tecnológicos y organizativos. Sin embargo, también es necesario obtener información del resto de las ramas para realizar análisis comparativos.

Dadas las limitantes enumeradas anteriormente, este tipo de información generada por las muestras específicas, son prácticamente incomparables entre sí a través de los cortes temporales, espaciales y sectoriales. ${ }^{8}$

Sin embargo, al tratar de sortear la mayoría de las limitantes de las muestras específicas se tienen que enfrentar restricciones demasiado fuertes. Desde luego, la principal es la restricción presupuestaria dado los altos costos de levantamiento de muestras que cumplan los requisitos deseados: que sean representativas, con amplia cobertura tempo-espacial y sectorial, exactitud y precisión, etcétera. Además existen restricciones de orden técnico y operativo que algunas veces dificultan cumplir con los requisitos. En concreto, en su experiencia, Patricia A. Wilson señala que obtener una muestra representativa de plantas maquiladoras resultó prácticamente imposible, en parte por la carencia de un conocimiento completo del universo y en parte porque un número de gerentes de plantas no concedieron entrevistas exhaustivas y completas.

Finalmente, la elección del tipo de muestra y del sistema de muestreo dependerá de: 1) el propósito de la investigación, 2) la naturaleza del fenómeno investigado, 3 ) el modo de inferencia adoptado, 4) de la exactitud, precisión y eficiencia requerida y 5 ) de los costos. Los cuatro primeros factores determinan la validez del procedimiento y los datos obtenidos, mientras el último factor determina la factibilidad.

Aquel enfoque de toma de datos que use correctamente una técnica, debe preguntarse al comienzo de la investigación si sus credenciales son adecuadas para el logro de un objetivo determinado (Harvey, 1983:362-371).

\section{Instrumentos de medición}

Por el tipo de instrumentos utilizados para tratar de clasificar, medir y detectar la magnitud y dirección del cambio tecnológico, se identifican claramente dos tipos: 1) los instrumentos directos como son la aplicación de encuestas o entrevistas que tratan de recoger variables e indicado-

8 La incapacidad de hacer comparaciones se refuerza ya que las muestran están basadas sobre elementos diferentes. Elementos tales como la población objetivo, la metodología, los alcances tempo-espaciales y sectoriales, el tiempo de levantamiento, etc. 
res más pertinentes a la particularidad, objetivos e hipótesis del estudio; y, 2) los instrumentos indirectos como son los modelos económico-matemáticos que tratan, primero, aportar un marco analítico de los factores y causas del cambio tecnológico y, además, sortear los problemas de la falta de pertinencia de las variables de las estadísticas oficiales.

Cada tipo de instrumentos tiene sus ventajas y limitantes de acuerdo a los criterios de evaluación que se utilicen. Para fines del presente estudio, la evaluación de los instrumentos utilizados por los estudios mostrados en el cuadro 2 se hará sobre la guía de la siguiente pregunta: $\iota q u e ́$ instrumento es el "más adecuado y preciso" para cumplir con el propósito planteado, es decir, medir el proceso de cambio tecnológico en la IME, tomando en cuenta que el cambio tecnológico es un proceso que se manifiesta y se capta más claramente en el mediano y largo plazo?

\section{a) Instrumentos directos}

Dada la diversidad de variables e indicadores utilizados en las muestras específicas para tratar de medir los cambios tecnológicos y organizativos, no es posible entrar en mucho detalle, simplemente se partirá del supuesto de que la pertinencia es adecuada, es decir, que la variable o indicador mide la propiedad o el atributo que se desea cuantificar.

Suponiendo que la aplicación de encuestas y entrevistas se realicen sobre muestras estadísticamente representativas, con cobertura espacial y sectorial adecuada, aún cumpliendo con estos requisitos se presenta una seria limitante; las muestras no tienen continuidad y comparabilidad en el tiempo. Por tanto, se dificulta la realización del análisis dinámico requerido por la naturaleza del proceso de cambio tecnológico.

\section{b) Instrumentos indirectos}

Dado que las estadísticas oficiales no reportan medidas directas de variables que den cuenta del proceso de incorporación de las nuevas tecnologías y formas de organización en la IME, se recurre a la aplicación de una serie de modelos económico-matemáticos con el objetivo de estimar un conjunto de indicadores que arrojan información más pertinente sobre la magnitud, dirección y tipo de cambio tecnológico experimentado en la IME a través del tiempo,

9 Los principales indicadores estimados por los estudios del cuadro 2 que utilizaron esta metodología son 1) la productividad media del trabajo, 2) la relación capital/trabajo, 3) el cambio tecnológico neutral, 4) el cambio tecnológico incorporado al trabajo, 5) la elasticidad de sustitución factorial y 6) el parámetro de escala, por mencionar los más comunes. 
Es decir, permiten no sólo la medición del cambio tecnológico, sino evaluar si dicho cambio en el tiempo es de carácter estructura. ${ }^{10}$

Efectivamente, estos instrumentos son más adecuados para los objetivos de medición y clasificación del cambio tecnológico. Sin embargo, presentan problemas de precisión debido principalmente a las limitantes de la estadística oficial de la que se alimentan: 1) insuficiente nivel de desagregación que no permite determinar cómo y dónde se está dando el cambio tecnológico, 2) series temporales relativamente cortas y 3) carencia de información para variables claves de los modelos. ${ }^{11}$ Para sortear este último problema, a los modelos originales se les debe realizar una serie de transformaciones estadístico-matemáticas y agregar ciertos supuestos. Desde luego, la transformación se paga con algún grado de sacrificio de la robustez del dato.

\section{CONCLUSIONES: ¿CAMBIO TECNOLÓGICO ESTRUCTURAL EN LA IME?}

El hecho de asegurar que los estudios partieran de un conjunto de objetivos e hipótesis similares fue con la finalidad de captar las discrepancias y varianzas que surgen en la utilización de los métodos y evidencias empíricas utilizadas. A continuación corresponde hacer el análisis de los resultados y las conclusiones a las que se arriban finalmente. La evaluación se hará sobre la base de la siguiente pregunta: ¿ existe un cambio de carácter estructural en el nivel tecnológico de la industria maquiladora de exportación en México?

En el cuadro 3 se muestran los principales resultados y conclusiones a los que arribaron cada uno de los estudios seleccionados. Inmediatamente salta a la vista que los estudios llegan a conclusiones y resultados un tanto diferentes. El argumento central del trabajo es que lo anterior es el resultado mismo de la gran heterogeneidad de las plantas maquiladoras, pero también es el resultado de las diferencias de los estudios en cuanto a instrumentos de medición y evidencias empíricas utilizadas.

Una primera imagen de la situación tecnológica de las plantas maquiladoras es mostrada en los resultados obtenidos por Patricia A. Wilson. Del total de las plantas maquiladoras incluidas en la muestra, sólo el $18 \%$

${ }^{10}$ Un tratamiento de carácter estructural del cambio tecnológico en la IMB es presentado por J. Alberto Godínez Plascencia en; "El Cambio Tecnológico en la Industria Maquiladora Electrónica y el Efecto en el Empleo" 1990.

11 Variables claves tales corno los volúmenes físicos de la producción e insumos, flujos y stocks de inversiones (capital), precios de productos y factores, etc. (Para mayores detalles véase a Bernardo González-Aréchiga, 1988). 
se puede considerar que están utilizando las estrategias competitivas postfordistas. ${ }^{12}$ Sin embargo, los segmentos de mayor crecimiento son las plantas maquiladoras de manufactura fordista $(35 \%)$ - especialmente las ubicadas en el interior del país - y las plantas de mano de obra intensiva ubicadas a lo largo de la frontera norte (44\%). Además, Wilson afirma que "el postfordismo encontrado en las plantas maquiladoras es una caricatura en comparación con el que ha sido experimentado en las economías desarrolladas" (Wilson, 1989).

Los resultados anteriores son reforzados por las conclusiones a las que llegan Flor Brown y Lilia Domínguez (1989:215-223). Quienes afirman que en la industria maquiladora existe una heterogeneidad tecnológica entre plantas. La heterogeneidad también se da en el interior de las plantas que cuentan con las nuevas tecnologías (coexiste la automatización con procesos intensivos en mano de obra). Concluyen sefialando que el proceso de introducción de las nuevas tecnologías en la IME es reciente, parcializado a través del proceso de producción y concentrado en las ramas electrónica y automotriz.

Para el caso de la rama electrónica, y a través de una muestra de mayor tamaño y cobertura geográfica, Mertens y Palomares, con cierta anticipación, llegan a resultados y conclusiones semejantes a los de Brown y Domínguez en cuanto a la identificación de las características del proceso de introducción de las nuevas tecnologías en la IME (Mertens y Palomares, 1985:170-198).

Por su parte -y a través de diversas investigaciones-, Bernardo González y J. Carlos Ramírez encuentran que la industria maquiladora está pasando por un doble proceso de creciente sofisticación y creciente dualismo tecnológico, con un crecimiento mayor de las empresas ubicadas en la parte baja de la escala tecnológica. Así, sefialan que es posible esperar en la década de los noventas que la IME crezca en dos vertientes: 1) el tradicional ensamble simple se mantendrá e incluso crecerá en la medida en que la automatización y los salarios comparativos de México lo permitan, y 2) se fortalecerá la tendencia hacia una nueva maquiladora más intensiva en capital y en la utilización de procesos completos de manufactura. Finalizan pronosticando que de esta forma es de esperarse que se mantenga e incluso se amplíe el dualismo tecnológico (González-Aréchiga y Ramírez, 1990:17-46).

Al respecto, Jorge Carrillo implícitamente no comparte la tesis del dualismo tecnológico al afirmar que "más que homogeneidad en el uso de

12 Una planta postfordista debe entenderse como aquélla que utiliza intensivamente los avances de la automatización flexible y las nuevas formas de organización del trabajo y la producción. 
CUADRO 3. Resultados y conclusiones de los principales estudios sobre el cambio tecnológico en la industria maquiladora.

Autor Resultados y conclusiones

Sanderson En 1984, del valor total del sector electrónico maquilador, la fabricación de componentes ocupó el 32\%, ensambles externos 13\%, 1987 subsistema de ensamble 32\% y los sistemas de ensamble 23\%. Asimismo, cada subsector tomará para automatizar el proceso de producción a un $80 \%$ de $5,7,10-15$ años respectivamente.

Cerca de 30 mil empleos y 450 millones de dólares de ingreso podrían ser afectados en los próximos 5 años (1985-1990) si la automatización continúa a la tasa estimada.

González La industria se compone, por un lado, de plantas modernas que se instalaron con procesos productivos complejos o que han 1988 evolucionado incorporando mejoras tecnológicas importantes y, por el otro, se compone de plantas tecnológicamente poco sofisticadas que recurren principalmente al ensamble simple. Esta dualidad tecnológica se manifiesta no sólo a nivel de la rama industrial sino también a nivel de la empresa e introduce una enorme fuente de varianza en el análisis económico de la $\mathrm{MME}$.

Es decir, la rME está pasando por un doble proceso de creciente sofisticación y creciente dualismo tecnológico, con un crecimiento mayor de las empresas ubicadas en la parte baja de la escala tecnológica.

González Las evidencias sustentan la hipótesis de que está surgiendo una nueva MME que, siendo altamente tecnificada, incorpora procesos y Ramírez 1988 completos de manufactura y no sólo de ensamble simple, pero que no refleja su productividad necesariamente en un mayor valor agregado y en un aumento de salarios en México.

Mertens

y Palomares

1988

Coexistencia de procesos tradicionales con procesos tecnológicos avanzados.

La introducción de las nuevas tecnologías dentro de algunos procesos productivos no se da de manera homogénea, sino a través de un proceso intermitente, parcial y en diferentes momentos.

El común denominador de todas las empresas electrónicas visitadas consiste en tener una estrategia que logre la formación de un nuevo tipo de trabajador bajo actitudes y comportamientos diferentes a los hasta ahora requeridos.

Wilson

La producción flexible y los bajos salarios no son factores de localización excluyentes.

1989

Sin embargo, el postfordismo encontrado en las plantas maquiladoras es una caricatura en comparación con el que ha sido experimentado en las economías desarrolladas.

- Sólo una minoría de plantas maquiladoras (18\%) se puede considerar que están utilizando las estrategias competitivas postfordistas. 
Los segmentos de mayor crecimiento son las plantas maquiladoras de manufactura fordista (35\%) - especialmente en el interiory las plantas ensambladoras de mano de obra intensiva (44\%) ubicadas a lo largo de la frontera norte.

Brown y En la IMR existe una gran heterogeneidad tecnológica entre plantas.

Domínguez La heterogeneidad tecnológica existe también en el interior de las plantas que cuentan con las nuevas tecnologías (coexiste la 1989 automatización con procesos intensivos en mano de obra).

La incorporación de las nuevas tecnologías es reciente, parcial y concentrada.

Carrillo Más que homogeneidad en el uso de ciertas tecnologías, ola existencia de procesos dicotómicos o duales, existen diferentes formas 1989

Godínez de producción y cambios tecnológicos.

1990

No existe evidencia de un cambio de carácter estructural en el nivel tecnológico de la IME. Tampoco es clara la inminencia de un desempleo masivo causado por motivos tecnológicos.

Es muy posible que el cambio tecnológico sea de reciente incorporación, parcializado a través del proceso de producción, concentrado en la rama electrónica y automotriz (incluso concentrado en ciertos productos) y centralizado en las grandes empresas. Los bajos salarios, cuantitativa, cualitativa y relativamente han perdido importancia como factor de localización y como política eficiente para generar empleos en la IME.

Mungaray La ventaja comparativa de la IME en México tradicionalmente ha estado en el abaratamiento de la fuerza de trabajo y no en el 1990 incremento de la productividad lograda con su estructura técnico-productiva.

Sin embargo, se puede decir que desde 1982 la ME se encuentra en una transición en busca de mayores niveles de rentabilidad por la vía del incremento del excedente sobre nuevas bases tecnológicas, con la tasa de ganancia positiva coyuntural proveniente del uso intensivo de la fuerza de trabajo, en vías de financiar, parcialmente por supuesto, dicha transición.

González Puede esperarse que el sector maquilador de la próxima década crezca en dos vertientes: 1) el tradicional ensamble simple se y Ramírez mantendrá e incluso crecerá en la medida en que la automatización y los salarios comparativos de México lo permitan, 2) se fortalecerála tendencia hacia una nueva maquiladora más intensiva en capital y la utilización de procesos completos de manufactura. Debe esperarse que se mantenga, e incluso se amplíe, el dualismo tecnológico en el que coexisten empresas que tienen una alta inversión por trabajador (mayor de 80 mil dólares) con empresas que operan con capital productivo solamente depreciado y valor nulo en libros (capital por trabajador menor a 5 mil dólares).

\section{FUENTE: Elaborado por en autor en base a la revisión de literatura teórica y empírica sobre la maquiladora.}


ciertas tecnologías, o la existencia de procesos dicotómicos o duales, existen diferentes formas de producción y cambios tecnológicos" (Carri1lo, 1990:105-131). La anterior afirmación da a entender que de cualquier manera existe un cambio tecnológico en la industria maquiladora, pero sólo expresado o diferenciado en la forma de llevarse a cabo.

J. Alberto Godínez (1990), utilizando las estadísticas oficiales y aplicando métodos económicos-matemáticos para realizar un tratamiento estructural de los cambios en los indicadores y parámetros tecnológicos de la IME, llega a la conclusión de que si bien ha habido un proceso relativamente rápido en la incorporación de las nuevas tecnologías y formas de organización, no ha sido de tal gradoa como para hablar ya de un cambio de corte estructural en el nivel tecnológico.

Después de revisar los principales resultados y conclusiones a los que llegan los estudios mostrados en el cuadro 3, puede decirse, en términos generales, que el esquema que más define al estado actual de la IME es la existencia de una heterogeneidad en la base tecnológica manifestada en una dualidad tecnológica, que se mantiene como estructura y como proceso tendencial. Este esquema se reproduce tanto entre plantas como al interior de las mismas, aquellas que cuentan con las tecnologías y formas de organización más avanzadas.

Antes de finalizar se tratará de dar una serie de recomendaciones técnico-metodológicas con el fin de mejorar y eficientar tanto los instrumentos de medición como las bases de datos sobre el cambio tecnológico en la IME y/u otras variables o características que se deseen conocer.

Para empezar, no está por demás la acertada crítica que realizan Bernardo González y J. Carlos Ramírez (1990) acerca de la pertinencia de cambiar de unidad de análisis teórica y empírica para estudiar el fenómeno de la subcontratación internacional en nuestro país. Esto es así porque las plantas maquiladoras incluidas en el programa maquilador constituyen cada vez más una unidad de análisis inadecuada para estudiar tal fenómeno, ya que han surgido y perfeccionado estructuras legales que facilitan la subcontratación fuera del tradicional esquema maquilador (PITEX, ALTEX, Industria Fronteriza, electrónica y automotriz).

Es necesario que los organismos oficiales encargados de generar y divulgar las estadísticas de la IME amplíen el espectro de información, incluyendo variables de importancia para la aplicación eficiente de los instrumentos económico-matemáticos. Variables tales como el volumen físico y precio de productos y factores, stocks y flujos de inversiones (capital), desagregación de la estructura de costos, coeficientes técnicos para determinar la ingeniería económica, etc. Además, se requiere que de alguna manera la información sea lo más desagregada 
posible en cuanto a rama industrial, lugar de localización, periodos, tamaño de planta y, si fuera posible, por origen del capital.

El levantamiento de muestras representativas será un apoyo y complemento fundamental para el mejor conocimiento de la industria maquiladora y de la subcontratación internacional en nuestro país. El levantamiento de muestras se puede considerar el instrumento más eficaz para responder con mayor precisión y detalle al dónde, al cómo y con qué ritmo se está dando el proceso de incorporación de las nuevas tecnologías y formas de organización a la IME. Igualmente se pueden identificar los factores que impulsan o limitan el proceso y, a la vez, pueden obtenerse estimaciones sobre la magnitud y dirección de los posibles efectos.

Un aspecto que debe ser contemplado en el levantamiento de muestras, es la inclusión de variables o indicadores que traten de medir con mayor precisión el ritmo de incorporación tecnológica. Tal objetivo se puede asegurar al incluir variables, tales como la edad de la planta, edad y grado de obsolescencia de la tecnología ađquirida, fecha de iniciación y grado de avance en la aplicación de las nuevas formas de organización, las fases concretas donde se introducen los avances técnico-organizativos, planes y programas de adquisición y aplicación de nuevas tecnologías y formas de organización, adiestramiento y capacitación del trabajo, etc.

No deben descuidarse, claro está, las variables de corte general tales como la rama o incluso el producto o productos específicos, tamaño de planta, origen del capital, entre otras.

Además, es necesario incluir variables que den luz sobre aspectos que hasta el momento han permanecido desconocidos, o conocidos en forma parcial, e imprecisos. Aspectos tales como la situación técnico-organizativa teórica en la que se encuentra en determinado momento algún proceso o producto, facilidad para acceder a las nuevas tecnologías, principales mecanismos de transferencia internacional y local, factores o determinantes que impulsan o limitan a las empresas a introducir alta tecnología, principales efectos o implicaciones, etc.

Definitivamente, lo deseable es contar con muestras que cumplan con los requisitos de representatividad estadística, mayor cobertura tempoespacial y sectorial, adecuado nivel de desagregación, y pertinencia de las variables. Todo lo anterior con el objetivo de que se garantice un mínimo de validez y confiabilidad en la evidencia presentada. Sin embargo, el grado en que se cumpla con tales requisitos dependerá de los objetivos, exigencia y presupuesto del estudio en cuestión. 


\section{BIBLIOGRAFÍA}

BROWN, Flor y Domínguez, Lilia. 1989. "Nuevas tecnologías en la industria maquiladora de exportación". Comercio Exterior, vol.39, no. 3, marzo de 1989. Banco de Comercio Exterior, México.

CARRILLO, Jorge. 1990. "Calidad con consenso en las maquiladoras: ¿Asociación Factible?". Frontera Norte, vol. 1, no. 2, julio-diciembre de 1989. COLEF. Tijuana, B.C.

ERNST, Dieter. 1984. "Innovación, transferencia internacional de tecnología e industrialización en el tercer mundo: El caso de la microelectrónica". En: Isaac Minian (Compilador), Trasnacionalización y Periferia Semiindustrializada. Parte II. Libros del CIDE, México.

GODÍNEZ Plascencia, J. Alberto. 1990. "El cambio tecnológico en la industria maquiladora electrónica y el efecto en el empleo" (Tesis de Maestría). Maestría en Desarrollo Regional-Promoción 19881990, COLEF, julio de 1990, Tijuana, B.C.

GONZÁLEZ-ARÉCHIGA, Bemardo. 1987. "Estructura de la industria maquiladora de exportación: Un ensayo de interpretación y búsqueda de conceptos" (mecanoescrito), COLEF, julio, Tijuana, B.C.

GONZÁLEZ-ARÉCHIGA, Bernardo. 1989. "Fuentes del crecimiento y el cambio en la composición laboral de la industria maquiladora de exportación". En: Bernardo González-Aréchiga (Coord.), La Industria Maquiladora de Exportación Mexicana en los Sectores Electrónico y de Autopartes. Documentos de Trabajo, Colegio de la Frontera Norte-Fundación Friedrich Ebert. México.

GONZÁLEZ-ARÉCHIGA, Bernardo y J. Carlos Ramírez. 1989. "Productividad sin distribución: Cambio tecnológico en la industria maquiladora de exportación (1980-1986)". Frontera Norte, No. 1, vol. 1, enero-junio. COLEF, Tijuana, B.C.

GONZÁLEZ-ARÉCHIGA, Bemardo y J. Carlos Ramírez . 1990. "Perspectivas estructurales para la subcontratación en México". En: GonzálezAréchiga, Bernardo y Ramírez, J. Carlos (Comps.), Subcontratación y empresas trasnacionales: apertura y restructuración en la maquiladora. México. COLEF-Fundación Friedrich Ebert.

HARVEY, David. 1983. Teorías, leyes y modelos en geografia. Madrid, España, Alianza Universidad, Textos.

INEGI. 1991. Estadisticas Básicas de la Industria Maquiladora de Exportación (circulación interna). INEGI, México.

JUNNE, Gerd. 1986. "Nuevas tecnologías: Una amenaza para las exportaciones de los países en desarrollo", Universidad de Amsterdam, 
Países Bajos. En Secretaría de Trabajo y Previsión SocialPNUD/OrT, Revolución tecnologica y empleo. Efectos sobre la división internacional del trabajo. México, STPS-PNUD/OTT.

KAPLINSKY, Raphael. 1988. "Modelos cambiantes de ubicación industrial y de competencia internacional: El papel de las empresas trasnacionales (ETN) y el impacto de la microelectrónica". En: Isaac Minian (Compilador), Cambio Estructural y Producción de Ventajas Comparativas. Libros del CIDE, México.

MERTENS, Leonards y Palomares, Laura. 1985. "El surgimiento de un nuevo tipo de trabajador en la industria de alta tecnología: El caso electrónico". En Esthela Gutiérrez Garza (Coord.), Testimonios de la Crisis: 1. Reestructuración Productiva y Clase Obrera. México, Siglo XXI-UNAM.

MUNGARAY L., Alejandro. 1990. Crisis, automatización y maquiladoras. UABC, Mexicali, B.C.

RADA F., Juan, 1983. "La microelectrónica, la tecnología de la información y sus efectos en los países en vías de desarrollo". Jornadas 97. El Colegio de México, México.

RAMÍREZ, J. Carlos y Bernardo González-Aréchiga. 1989. "Los efectos de la competencia internacional en el funcionamiento de la industria maquiladora de exportación en México". Frontera Norte, No. 2, vol. 1, julio-diciembre, COLEF Tijuana, B.C.

SANDERSON, Susan W. 1987. "Automated Manufacturing and Offshore Assembly in Mexico". En: Cathryn L. Thorup, the United States and Mexico: Face to Face withNew Technology. U.S. (Third World Policy Perspectives No. 8), Overseas Development Council, Washington, D.C.

WILSON, Patricia A. 1989. The New Maquiladoras: Flexible Production In Low Wage Regions. University of Texas at Austin. Community and Regional Planning. Working Papers Series, no.9, abril. 